\title{
Autoimmune Thrombocytopenia in SLE and COVID-19
}

\author{
Yeremia Suryo Pratama ${ }^{1}$, Riska Pradiptakirana ${ }^{1}$, Azkia Rachmah $^{1}$, Nurhasan Agung Prabowo $^{2}$ \\ ${ }^{1}$ Faculty of Medicine, Sebelas Maret University, Surakarta, Indonesia \\ ${ }^{2}$ Internal Medicine Department, Faculty of Medicine, Sebelas Maret University Hospital, Surakarta, Indonesia
}

Received: 06/09/2021

Accepted: 05/10/2021

Published: 03/11/2021

How to cite this article: Pratama YS, Pradiptakirana R, Rachmah A, Prawobo NA. Autoimmune thrombocytopenia in SLE and COVID-19. EJCRIM 2021;8: doi:10.12890/2021_002863.

Conflicts of Interests: The authors declare there are no competing interests.

Acknowledgements: We sincerely send our gratitude to our patient that was involved in this study. We also warmly appreciate Sebelas Maret University Hospital and its staff that provided treatment to our patient and provided us with the necessary data for our case report.

This article is licensed under a Commons Attribution Non-Commercial 4.0 License

\section{ABSTRACT}

Thrombocytopenia and hypercoagulopathy are haematological abnormalities commonly seen in individuals with coronavirus disease 2019 (COVID-19) and systemic lupus erythematosus (SLE). The difficulty arises when the patient has both diseases concurrently. The clinician should be able to comprehend the pathophysiology of these patient abnormalities in order to provide the best treatment possible. We present a case of a 20-year-old female COVID-19 patient with a history of SLE who had thrombocytopenia but normal D-dimer results. Our analysis revealed that the thrombocytopenia may have been caused by a relapse of lupus, not by COVID-19 infection. In this case, glucocorticoids were the primary therapy and produced excellent results.

\section{LEARNING POINTS}

- The pathophysiology of thrombocytopenia in a patient with concurrent COVID-19 and SLE should not always be associated with platelet consumption.

- Low-dose glucocorticoids should be administered, with infection risk and comorbidities taken into consideration.

- Glucocorticoid therapy may result in a delay in viral clearance.

\section{KEYWORDS}

COVID-19, hypercoagulopathy, SLE, thrombocytopenia

\section{INTRODUCTION}

Thrombocytopenia is a common haematological finding in patients with coronavirus disease 2019 (COVID-19) and systemic lupus erythematosus (SLE). It is associated with an increased risk of death, regardless of bleeding events ${ }^{[1]}$. It is critical to understand the mechanism underlying thrombocytopenia whether is caused by COVID-19 or SLE in order to provide appropriate treatment, which should be determined on an individual basis, taking infection risk and comorbidities into account. In this article, we report a case of a patient presenting with thrombocytopenia who was subsequently found to have COVID-19 and SLE. 


\section{CASE DESCRIPTION}

A 20-year-old female came to the emergency department with 2 days of fever and coughing. The patient admitted a history of SLE but denied any previous bleeding events. The clinical examination revealed no signs or symptoms of bleeding. Chest $x$-ray (CXR) represented bronchopneumonia in both lungs (Fig. 1). A complete blood count (CBC) was performed and showed a platelet count of $11 \times 10^{3} / \mu$ l. A positive result for reverse transcription-polymerase chain reaction (RT-PCR) testing for SARS-CoV-2 confirmed a COVID-19 diagnosis. Additional laboratory and ANA profile tests were also performed on the patient (Table 1). The patient received an IV dose of methylprednisolone 30 $\mathrm{mg} / 8$ hours. The CBC on the following days showed a gradual increase in the platelet count (Fig. 2). The patient was stable and discharged home to follow an independent isolation protocol on the 14th day of hospitalization due to the patient's RT-PCR test still showing a positive result.

\begin{tabular}{|l|l|l|}
\hline General & Labs at admission & Peak/nadir values \\
\hline Haemoglobin $(\mathrm{g} / \mathrm{dl})$ & 9.2 & 8.5 (Day 2) \\
\hline Platelets $\left(10^{3} / \mathrm{\mu l}\right)$ & 11 & 11 (Day 1$)$ \\
\hline WBC $\left(10^{3} / \mathrm{\mu l}\right)$ & 6.42 & 4.99 (Day 2) \\
\hline Creatinine $(\mathrm{mg} / \mathrm{dl})$ & 0.65 & \\
\hline Urea $(\mathrm{mg} / \mathrm{dl})$ & 10 & \\
\hline Hs-CRP $(\mathrm{mg} / \mathrm{dl})$ & $<0.001$ & \\
\hline D-dimer & 276 & \\
\hline INR & 0.88 & \\
\hline aPTT & 31.8 & \\
\hline PT & 12.90 & - \\
\hline PCNA & & +++ \\
\hline Ro-52 recombinant (52) & & ++ \\
\hline SSA native & & \\
\hline Centromere B & & \\
\hline
\end{tabular}

Table 1. Results of laboratory and immunology investigations in the patient

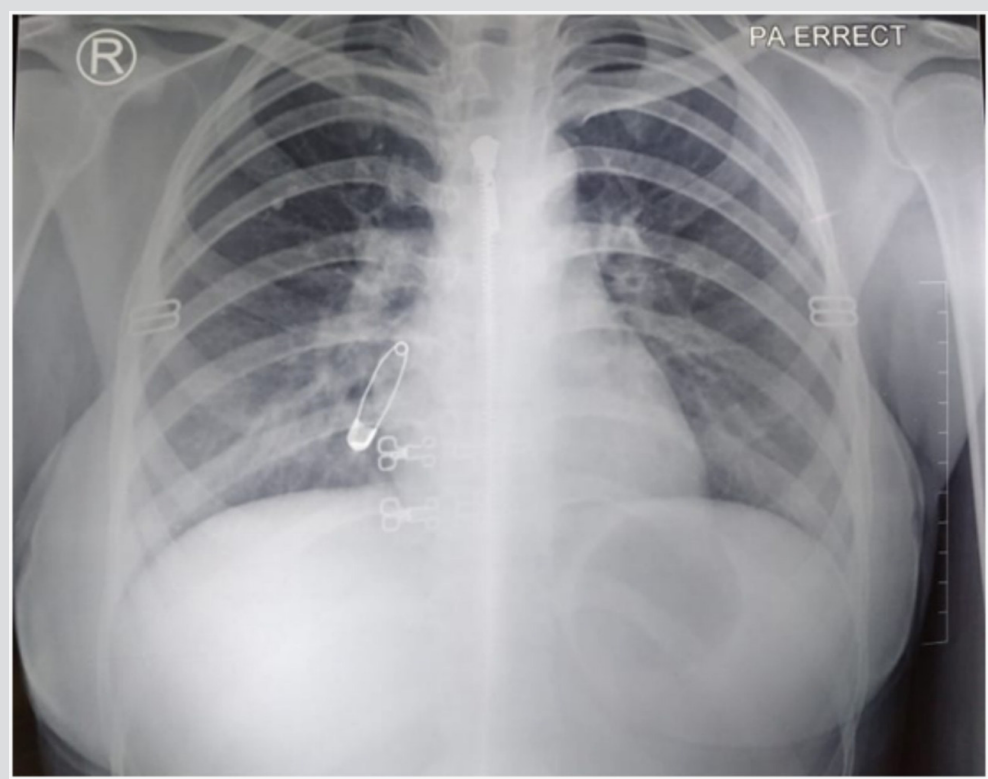

Figure 1. The chest $x$-ray showed multiple infiltrates in both lung fields depicting bronchopneumonia 


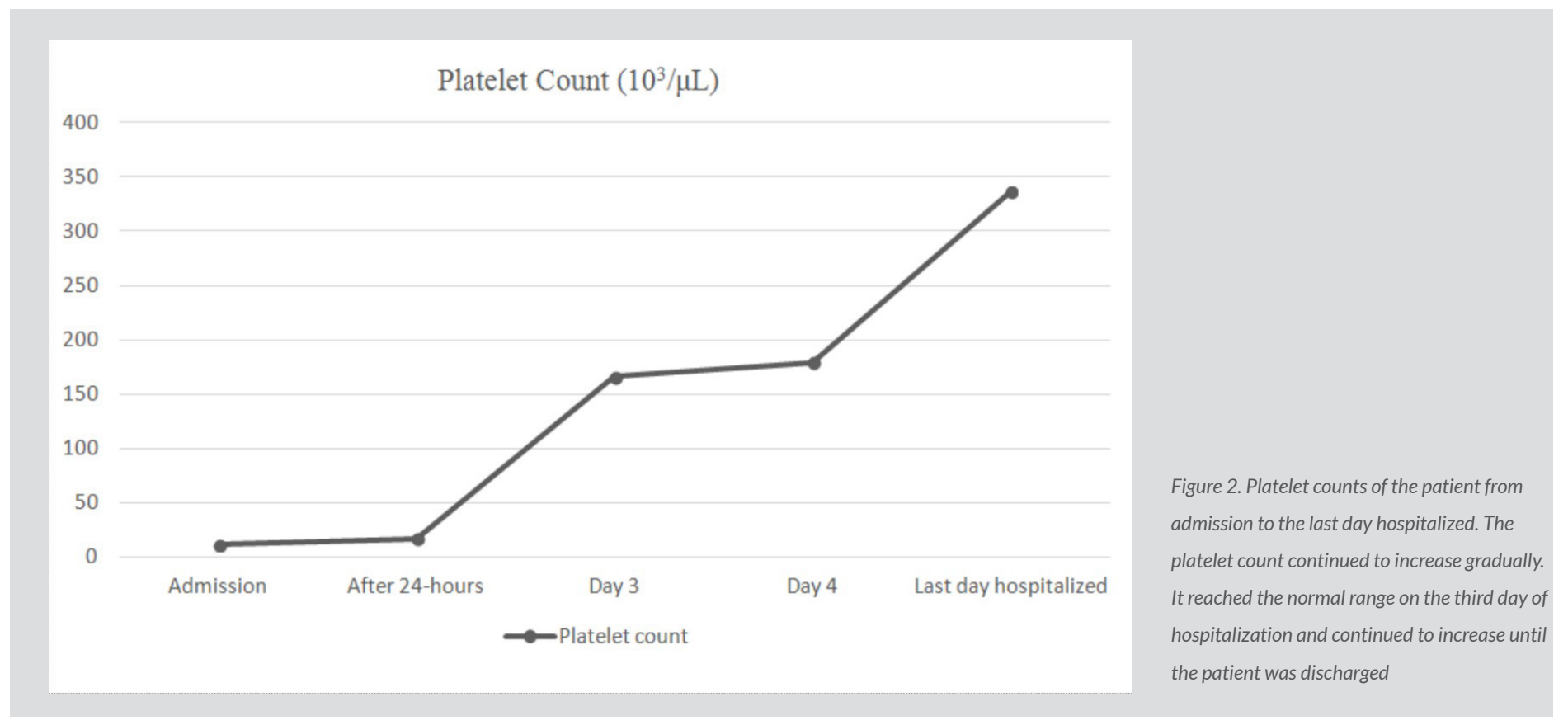

\section{DISCUSSION}

Thrombocytopenia, defined as a platelet count of less than $150 \times 10^{3} / \mu \mathrm{l}$, is 1 of the most common laboratory parameter abnormalities in patients with COVID-19. It was thought that the aetiology underlying this change was orchestrated by various mechanisms, such as direct inhibition of platelet synthesis in bone marrow, increased platelet destruction by the immune system and increased platelet aggregation in the lungs ${ }^{[2]}$. Elevated D-dimer, defined as a D-dimer count of more than $500 \mathrm{mcg} / \mathrm{l}$, was recently established as a risk factor for more critically ill patients and related to increased mortality ${ }^{[3,4]}$, that prompted monitoring of D-dimer to predict a severe case ${ }^{[5]}$.

Thrombocytopenia is also commonly found in SLE patients. The inflammatory process in SLE could increase the risk of clotting. Several studies have found an elevation in D-dimer in SLE patients who are at risk of thrombosis. This, however, is not the only cause of thrombocytopenia in SLE ${ }^{[6]}$. The circulating platelet destruction in SLE was also centrally attributed to the mechanism involving autoantibodies in the platelet membrane. Other autoantibodies detected in SLE cases, such as an antiphospholipid antibody or antibody towards thrombopoietin (TPO), are also thought to affect thrombocytopenia by other means ${ }^{[7]}$. Apoptosis of megakaryocytes in the bone marrow by autoantibodies causes thrombocytopenia in lupus. This condition does not affect the coagulation system, and D-dimer is not affected.

Our COVID-19 patient presented with thrombocytopenia but without concomitant elevated D-dimer. As the D-dimer itself is a byproduct of fibrinolysis, these findings may show that the thrombocytopenia in COVID-19 patients with SLE is not always attributable to increased consumption of platelets due to thrombosis in damaged lung tissue, as reported in a previous study ${ }^{[8]}$. We speculate that this unusual presentation was predominantly set in motion by the autoantibody activity against circulating platelets, coupled with COVID19-related inhibition of platelet production and increased platelet destruction. A study in Wuhan in 178 COVID-19 patients suggested that thrombocytopenia was associated with elevated D-dimer and abnormal coagulation, and was associated with mortality. Therefore, if D-dimer levels are normal, it indicates that there is no thrombus formation which is a sign of the main pathogenesis of COVID-19. Hence, the possible cause is bone marrow suppression due to lupus autoantibodies ${ }^{[9]}$.

Long-term steroid usage, as in SLE patients, may contribute to the SARS-CoV-2 infection rate. Despite this, sudden glucocorticoid cessation appears to be a significant cause of flare in such patients, and the decision to stop the treatment should be examined on an individual basis, taking individual infection risk and comorbidities into account ${ }^{[10]}$. Furthermore, steroid administration is still questionable in COVID-19. A study showed that low-dose glucocorticoid is favourable in increasing survivability in COVID-19 patients ${ }^{[11]}$.

In our case, we administered glucocorticoids to our patient due to the numerous benefits recognized in previous studies [10, 11]. The antiinflammatory effects of glucocorticoids can help reduce alveolar exudation and may also be useful in preventing and alleviating cytokine storms. In addition, the glucocorticoid could also control the disease progression of SLE and could mitigate its flare. Despite the benefits, glucocorticoids may slow viral clearance in COVID-19 patients ${ }^{[9]}$, as demonstrated in our patient, who retained a positive PCR result despite her resolving condition. 
In conclusion, the pathogenesis of thrombocytopenia in COVID-19 with SLE should not be always associated with increased platelet consumption, especially when there is limited laboratory parameter evidence, such as normal D-dimer or normal coagulation time. In this study, we speculate that there are several mechanisms involving COVID-19 and SLE conditions that could cause thrombocytopenia that are hypothetically more prominent than the platelet consumption or thrombosis-related mechanism. However, the scarcity of data regarding the D-dimer and platelet count status in patients with SLE and COVID-19 means our finding cannot be substantiated. Because of this limitation, we implore more investigation on a wider population of COVID-19 and SLE patients, that also assesses whether elevated D-dimer and thrombocytopenia are related in patients with both SLE and COVID-19. Our study also supports the usage of glucocorticoids in the treatment of concomitant COVID-19 and SLE, although this decision should be determined on an individual basis, taking infection risk and comorbidities into account ${ }^{[9]}$. Nevertheless, we support and implore care providers to always check for possible coagulopathy by assessing D-dimer levels and coagulation time in thrombocytopenia in COVID-19, especially among patients with a history of SLE. Further immunology investigation, such as screening for possible antiphospholipid syndrome (APS), also seems useful to predict the prognosis.

\section{REFERENCES}

1. $\quad$ Yang X, Yang Q, Wang Y, Wu Y, Xu J, Yu Y, et al. Thrombocytopenia and its association with mortality in patients with COVID-19. J Thromb Haemost 2020;18(6):1469-1472. Xu P, Zhou Q, Xu J. Mechanism of thrombocytopenia in COVID-19 patients. Ann Hematol 2020;99(6):1205-1208.

3. Vidali S, Morosetti D, Cossu E, Luisi MLE, Pancani S, Semeraro V, et al. D-dimer as an indicator of prognosis in SARS-CoV-2 infection: a systematic review. ERJ Open Res 2020;6(2):00260-2020.

4. Rostami M, Mansouritorghabeh H. D-dimer level in COVID-19 infection: a systematic review. Expert Rev Hematol 2020;13(11):1265-1275.

5. Han H, Yang L, Liu R, Liu F, Liu F, Wu KL, et al. Prominent changes in blood coagulation of patients with SARS-CoV-2 infection. Clin Chem Lab Med 2020;58(7):1116-1120.

6. Wu H, Birmingham DJ, Rovin B, Hackshaw KV, Haddad N, Haden D, et al. D-dimer level and the risk for thrombosis in systemic lupus erythematosus. Clin J Am Soc Nephrol 2008;3(6):1628-1636.

7. Sufian ABMA, Kashem M, Biswas S. Pattern of hematological manifestations in patients with systemic lupus erythematosus attending in a tertiary care hospita. $J$ Med 2017;18(2):86-91.

8. Hayden A, Vyas-Lahar A, Rella V, Rudinskaya A. Severe refractory thrombocytopenia in a woman positive for coronavirus disease 2019 with lupus and antiphospholipid syndrome. Lupus 2020;29(11):1472-1474.

9. Bao C, Tao X, Cui W, Yi B, Pan T, Young K, et al. SARS-CoV-2 induced thrombocytopenia as an important biomarker significantly correlated with abnormal coagulation function, increased intravascular blood clot risk and mortality in COVID-19 patients. Exp Hematol Oncol 2020;9:16.

10. Zucchi D, Tani C, Elefante E, Stagnaro C, Carli L, Signorini V, et al. Impact of first wave of SARS-CoV-2 infection in patients with systemic lupus erythematosus: weighting the risk of infection and flare. PLoS One 2021;16:e0245274.

11. RECOVERY collaborative group et al. Dexamethasone in hospitalized patients with Covid-19. N Engl J Med 2021;384(8):693-704. 